Review article

\title{
Meta-analysis and systematic review for the treatment of perpetrators of intimate partner violence
}

\author{
Günnur Karakurt ${ }^{\mathrm{a}, *}$, Esin Koç ${ }^{\mathrm{b}}$, Ezgi Elif Çetinsaya ${ }^{\mathrm{b}}$, Zozan Ayluçtarhan ${ }^{\mathrm{b}}$, Shari Bolen ${ }^{\mathrm{c}}$ \\ ${ }^{a}$ Case Western Reserve University, University Hospitals Cleveland Medical Center, United States \\ ${ }^{\mathrm{b}}$ Bilkent University, Turkey \\ ${ }^{\mathrm{c}}$ Center for Health Care Research and Policy CWRU at the MetroHealth Medical Center, And Department of Medicine, MetroHealth Medical Center, United States
}

\section{A R T I C L E I N F O}

\section{Keywords:}

Meta-analysis

intimate partner violence

domestic violence

perpetrator

batterer intervention programs

recidivism

\begin{abstract}
A B S T R A C T
Aim of this study is to investigate the effectiveness of different batterer intervention programs in reducing violence for male IPV perpetrators. The Cochrane Handbook for Systemic Reviews of Interventions guidelines for the process of conducting systematic reviews and meta-analysis were followed. Pooled together, overall these various intervention programs are effective in reducing violence for male perpetrators of IPV comparing post to pre-intervention [(pooled estimate $=-0.85$; 95\% Confidence Interval (CI) (-1.02 to -0.69)]. Exploratory subgroup analysis revealed that incorporating substance abuse or trauma components to the interventions yielded better results (substance abuse: $\mathrm{CI}=-3.20$ to -1.08 and trauma: $\mathrm{CI}=-2.63$ to -0.30 ) as compared to programs that did not have these components. Gender-role based batterer intervention programs yielded mixed results. Analysis of the three controlled studies with 223 participants comparing batterer programs to a minimal control group showed mixed effects. In conclusion, treatment strategies that are addressing highly comorbid issues such as substance abuse and trauma issues may work more effectively in preventing violence.
\end{abstract}

\section{Introduction}

Intimate partner violence (IPV) is a detrimental social and public health problem with severe consequences (Black et al., 2011). Survivor victims suffer from mental and physical health problems in the short and long term (Smith et al., 2014; Karakurt et al., 2016). Family members and children who are exposed to violence also suffer from adverse health, social and developmental effects (Kelly \& El-Sheikh, 2013; Wathen \& Macmillan, 2013). It is estimated that approximately one in four women become a victim of severe violence at some point in their life regardless of their age, economic status, and ethnicity (Black et al., 2011).

Treatment approaches utilizing various formats, lengths, and theoretical orientations have been developed over the years to prevent and reduce IPV (Babcock et al., 2004; Gondolf, 2001; Murphy \& Meis, 2008). Most early interventions based on the Duluth model with the underlying feminist frameworks focused on men's utilization of power and control tactics (Pence \& Paymar, 1993). These programs were designed to prevent IPV by solely targeting male offenders (Adams \& Cayouette, 2002; Dobash et al., 1996). Research findings on batterer intervention programs indicate that the efficacy of these programs are inconsistent (Murphy et al., 2017; Stover et al., 2009). While Duluth like programs somewhat reduce violence, they do not work as well as expected (Babcock \& La Taillade, 2000; Miller et al., 2013). In many instances, there are small average effect sizes compared to minimal to no effect (Babcock et al., 2004; Feder \& Wilson, 2005; Miller et al., 2013). Furthermore, most of these programs have high dropout rates (Carney et al., 2006).

For many interventions, group therapy format was the most frequently utilized modality due to its cost-effectiveness and due to its flexibility in covering the wide range of psycho-educational components including anger management, problem-solving, and stress management (Babcock et al., 2007). The group format also provides opportunities for social intervening as well as positive peer influence (Yalom \& Leszcz, 2005). However, the group format was also found to be linked with unwanted consequences such as the normalization of aggressive behaviors (Murphy \& Meis, 2008) and antisocial peer influences among male perpetrators (Saunders, 2008).

Prior studies used various programs and techniques to reduce violence among offenders. These include a narrative analysis of painful memories of offenders based on psychodynamic approaches (Saunders, 1996), supporting offenders through group setting (Morrel et al., 2003), careful monitoring via probation and case management (Dunford, 2000a, 2000b). Many programs particularly Cognitive Behavioral

\footnotetext{
* Corresponding author.

E-mail address: gkk6@case.edu (G. Karakurt).
} 
Therapy (CBT) based interventions focus on transforming hostile cognitive biases, working on affect dysregulation, addressing skill-based deficiencies including assertiveness, communication, and problem-solving (Wexler, 2013). More action-oriented approaches, such as Acceptance and Commitment therapy focuses on replacing avoidance and denial with accepting issues, and committing a change in behavior (Zarling et al., 2015). Researchers also aimed to improve protherapeutic attitudes and preparedness to change in treatment by conducting motivational enhancement therapy prior to treatment to enhance engagement and active participation (Alexander \& Morris, 2008).

From violence researchers, there is a growing recognition and call for more rigorous outcome studies (Babcock \& La Taillade, 2000; Dunford, 2000a, 2000b; Gondolf, 2001; Miller et al., 2013; Feder \& Wilson, 2005). Findings are difficult to summarize due to high attrition rate, nonexperimental evaluation of the programs, lack of follow-ups, and reporting percent rate of recidivism as the main success indicator of the treatment programs (Dunford, 2000a, 2000b; Gondolf, 2004; Stover et al., 2009).

Given the deleterious effects of IPV on the wellness of families and society, there is an increasing need to understand what is effective in treating and preventing intimate partner violence based on goodquality evidence (Taft and Hegarty, 2010). In fact, a prior meta-analysis was conducted (Babcock et al., 2004; Miller et al., 2013) to investigate the effectiveness of batterer intervention programs. However, the effects of the meta-analysis were inconclusive due to a limited number of controlled studies and small effect sizes from the pooled research. In addition, new studies with hybrid approaches to treatment have become available since that meta-analysis was conducted. These approaches augmented their treatment based on the needs of their participants such as substance use treatment (Easton et al., 2007), traumafocused treatment (Taft et al., 2013) and use of motivational interviewing techniques (Morrel et al., 2003) for dealing with co-morbidity issues and high drop-out rates. The broad range of programs available for the treatment of intimate partner violence and the wealth of surveys and research studies that investigate the effectiveness of these programs provide an opportunity for systematic investigation of the success of different treatment programs. Therefore, we conducted a systematic review and meta-analysis aiming to: 1) systematically characterize the effectiveness of treatment programs for the perpetrators of IPV; and 2) explore potential differential effectiveness of specific programs.

\section{Methods}

\subsection{Identification of studies}

The Cochrane Handbook for Systemic Reviews of Interventions guidelines for the process of conducting high-quality systematic reviews and meta-analysis were followed for this study (Higgins \& Green, 2011). Prior to conducting a systematic review, the Populations, Interventions, Comparisons, Outcomes, Time, and Settings (PICOTS) of the studies that were going to be included in the meta-analysis were decided (Higgins \& Green, 2011). Once identified, screening of the literature based on PICOTS produce higher consistency across studies (Higgins \& Green, 2011). Therefore, the inclusion and exclusion criteria developed by using the PICOTS framework includes P: Physically abusive adult males, I: Active intervention is done to prevent and diminish IPV among males, for the current study screening and probation were not considered as an active intervention. C: pre-post test comparisons, O: Reduction in Violence, measured by the Conflict Tactic Scale, T: any time period, $\mathbf{S}$ : No setting limitations were applied.

We searched electronic databases from inception through August 2018. For each of the databases, our research contained keywords in addition to Medical Subject Headings (MESH) terms. For violence, we searched terms including: "domestic violence", "intimate partner violence", "perpetrator", "batterer". For interventions, we used terms including "therapy", "treatment", "intervention", "counseling", "prevention", "program", "curriculum", and "education". To maximize the number of studies, we used "OR" to combine these terms. Databases including PubMed, Ebsco/Host (Academic Search Complete; Eric, Family Studies Abstracts, Humanities \& Social Sciences Index Retrospective: 1907-1984 (H.W. Wilson), MEDLINE, Psychinfo, Psycharticles, and Cochrane Library were searched. Before finishing the search strategy, the research team consulted a university librarian. The research team also identified studies through a hand search during this time. Hand search articles included studies from a prior meta-analysis including Babcock et al., 2004 and Miller et al., 2013, all included studies as well as other relevant references related to the topic.

The titles, abstracts and the full articles were reviewed by two research assistants. Any disagreements raised were resolved at the research meetings by consensus. Team research meetings involved three research assistants and faculty advisor. The exclusion criteria for the title and abstract review were as follows: no original data, does not involve violent men, no peer review, duplicate, case study, and no subjects older than 18 years of age. We only included peer-reviewed studies since the peer review process provides an extra validation of the methods and results of the articles. Studies that are not directly assessing the effectiveness of the intervention for male perpetrators on clinically relevant violence reduction outcome were excluded. Using the recidivism rate raised concerns for clinical relevance as an outcome (Stover et al., 2009; Taft \& Hegarty, 2010). Therefore, we used the widely utilized conflict tactic scale as an outcome measure for this study to increase the clinical relevance of this outcome.

\subsection{Pre-Post comparisons}

For studies having more than one arm, intervention programs that target male perpetrators, and treatments for intimate partner violence were selected. If the study compared more than one intervention to reduce IPV, both interventions were selected for data abstraction. If studies reported follow up data, the closest time period to post-intervention was selected for data abstraction.

\subsection{Controlled Experimental Studies}

For control groups, we selected minimal controls (no-treatment control, a referral list, and pamphlets) as the comparison group since our goal is to understand the effectiveness of standard batterer intervention programs in treating IPV. We only abstracted data that includes the true control groups as a comparison, and did not pool data from studies which are utilizing (i) non-completers as control (ii) participants indicating different readiness to treatment as control (iii) participants from a different racial background as control. When multiple intervention groups were presented, we prioritized batterer interventions and group therapy interventions. For studies having more than one arm, most consistent intervention and control group to the other studies was used to enhance homogeneity across studies for the meta-analysis.

\subsection{Data abstraction}

Two team members serially abstracted data from included articles. Data were collected by one researcher, and then double checked by the other researcher for accuracy. We used standardized data abstraction forms to extract data on study design, duration, population, intervention, outcomes, and quality. Extracted data elements included violence reduction, type of treatment, number of sessions, curriculum and settings in which the treatment occurred. The conflict tactics scale (as a gold standard measure for intimate partner violence) and its derivatives such as the Revised Conflict Tactics Scale (CTS2) and Short version or modified version of the conflict tactic scale were used as benchmarks to measure the frequency of violent interactions. The differences in mean and standard deviation between intervention and control groups at pretest and post-test were extracted. This information provided a 
continuous outcome measure in changes in the frequency of male-tofemale violence. Most studies reported on total violence frequency with only a few stratifying on violence severity.

Whenever possible, we prioritized total violence, followed by severe violence as many of the studies conducted on court-ordered domestic violence treatment in severe cases.

\subsection{Quality of studies}

We assessed the quality of the studies in two ways. First, we used the Cochrane Intervention Studies Scale (Armijo-Olivo et al., 2012) for randomized control trials to assess study quality. We then used the Cochrane Risk of Bias tool to assess the additional risk of bias for these studies. Team members independently rated each study to assess the quality and then met to reach consensus together.

\subsection{Data Synthesis and Analysis}

Series of evidence tables were created using the data abstracted from the articles. When there were sufficiently homogenous data on intervention and control groups (minimal control), a meta-analysis was conducted (at least three studies). We summarized data in tables for a descriptive synthesis of the studies. We used table summaries and the Isquared statistic (I-squared scores $>50 \%$ indicate substantial heterogeneity) to determine whether there was sufficient homogeneity in treatment interventions, study populations, and outcomes for us to conduct meta-analyses (Higgins et al., 2003). Where there was sufficient homogeneity, MIX for meta-analysis software was used for analysis (Bax et al., 2006). We conducted stratified meta-analyses as well as subgroup comparisons to identify sources of heterogeneity when there were sufficient studies. Meta-analyses were conducted using a randomeffects model with the pooled mean difference between groups (DerSimonian \& Laird, 1986). The inverse variance method that takes into account the standard deviation, as well as the sample size, was utilized for calculating study weights.

\section{Results}

\subsection{Identification of the studies}

Electronic databases [PubMed $(\mathrm{N}=944)$, Cochrane Library ( $N=15)$, Ebsco/Host $(N=2633)$ with boolean search] in addition to Hand Searched articles $(\mathrm{N}=60)$ revealed a total of 2049 titles after duplicates were removed. Of the 2049 titles, 13 studies had pre-post data reported Figure 1. Three of these studies had a minimal control group as a comparison and two of them had a head to head comparisons. The main reasons for exclusion of articles were not having prepost data, not using CTS and not being relevant to our PICOTS.

\subsubsection{Characteristics of included studies}

The studies included have conducted various interventions to actively prevent and reduce IPV. Characteristics of the studies can be found in Table 1. The majority of the studies were conducted in the United States, one study was conducted in Canada, one study was conducted in Spain, and another study was conducted in Hong Kong. The majority of the U.S. studies were conducted in a Caucasian sample ranging from $40 \%$ to $83 \%$; two studies had a higher African American sample of $43 \%$ and $48 \%$. Most studies included young to middle-aged adult men (mean age ranged from 31.5 to 46.4 years). The majority of the interventions were delivered in the group therapy setting, while one study included individual therapy as a comparison group, and another study used telephone-delivered individual talk therapy. In the group therapy studies, the group therapy was delivered by a doctoral level therapist in 6 studies and a master level therapist in 4 studies. Duration of the treatment was ranging from 12 to 70 hours of treatment. Most of the studies have similar baseline CTS levels across studies except
Lawson 2009 which had higher CTS levels. Some studies included court-mandated participants (5 studies) while others included participants who were voluntarily seeking treatment (8 studies).

\subsubsection{Quality of the studies and Publication Bias}

Risk of bias for the current systematic review was investigated through selection bias, performance bias, detection bias, publication bias, and attrition bias. Fig. 2 present information regarding potential biases. In our study, funnel plot analyses demonstrate no clear indication of publication bias. Most studies had a low (69\%) or unclear (31\%) risk of selection bias. Potential sources for the risk of bias were identified as not providing enough information about inclusion criteria for the study, lack of randomization, and selection from a larger pool from a prior study. Performance bias was another potential bias source, with $62 \%$ of studies rated as low risk of bias and $38 \%$ of studies rated as unclear risk of bias. Performance bias was defined as whether there are systematic differences in how the treatment was delivered. Many treatment programs ( 9 studies) used manualized treatment to ensure participants in the study receive the same or similar treatments. Detection bias is based on how the outcome of the studies was measured, and whether there is any systematic difference in the outcome measure. In this study, we utilized the conflict tactic scale and deviates to assess the outcome. It is an already validated scale, with good psychometric qualities. Since the outcomes measured consistently across groups, we did not observe evidence of detection bias. Attrition bias occurs when there is a systematic difference between participants who complete the treatment versus non-completers due to certain characteristics of the sample. Attrition bias was found to be unclear (62\%) or high risk (15\%) of bias in most studies. In the 13 included studies, drop-outs ranged from $3 \%$ to $41 \%$ when reported with most $>24 \%$. Finally, reporting bias was found to be a low risk of bias for most studies (85\%). Reporting bias was unclear in $15 \%$ of the studies due to unclear reporting of completion rates as well as lack of detail in reporting of the results (Fig. 2).

\subsection{Effects based on pre-test post-test scores}

Our findings for pre and post comparison of the intervention studies indicate that IPV can be significantly reduced through programs designed for male perpetrators comparing post-test results to pre-test baseline levels of IPV $(\beta=-0.85,95 \%$ CI -1.02 to $-.69, p<.001$; see Fig. 3). The $\mathrm{I}^{2}$ statistic was equal to $87 \%$ with confidence interval $(95 \%$ CI) $80 \%$ to $91 \%$ indicating substantial heterogeneity. Heterogeneity can be due to the differences in treatments, sample populations or the study design (von Hippel, 2015). To further explore the differences in treatment, stratified analyses were conducted for different treatments including standard batterer intervention programs, Cognitive Behavioral Therapy (CBT) based approaches as well as augmented approaches. The forest plot for the subgroup analyses is shown in Fig. 4.

Exploratory subgroup analyses comparing stratified meta-analysis results suggest that treatment models augmented with substance abuse models yielded significantly more effective results in reducing violence for male perpetrators [mean difference $(\mathrm{md})=-2.14$, CI -3.20 to -1.08 ] Similarly, trauma augmented models yielded significantly more effective results at reducing violence for male perpetrators ( $\mathrm{md}=-1.47, \mathrm{CI}$ -2.63 to -0.30 ). On the other hand, studies using sex role or Duluth approaches produced mixed results at reducing violence for male perpetrators when compared to other treatments. Implementation of CBT, motivational or Standard Batterer Intervention (SBI) based approaches did not exhibit significant differences compared to each other, i. e., other therapy models. However, CBT treatment augmented with substance abuse treatments yielded more consistent results than the following augmented treatments: 1) CBT augmented with motivational enhancement treatments ( $\mathrm{md}=3.83$, CI 1.89 to 5.77 ), 2) motivational enhancement treatment augmented with substance abuse models (md $=1.94$, CI 0.41 to 3.48 ), and 3) CBT based treatments without 


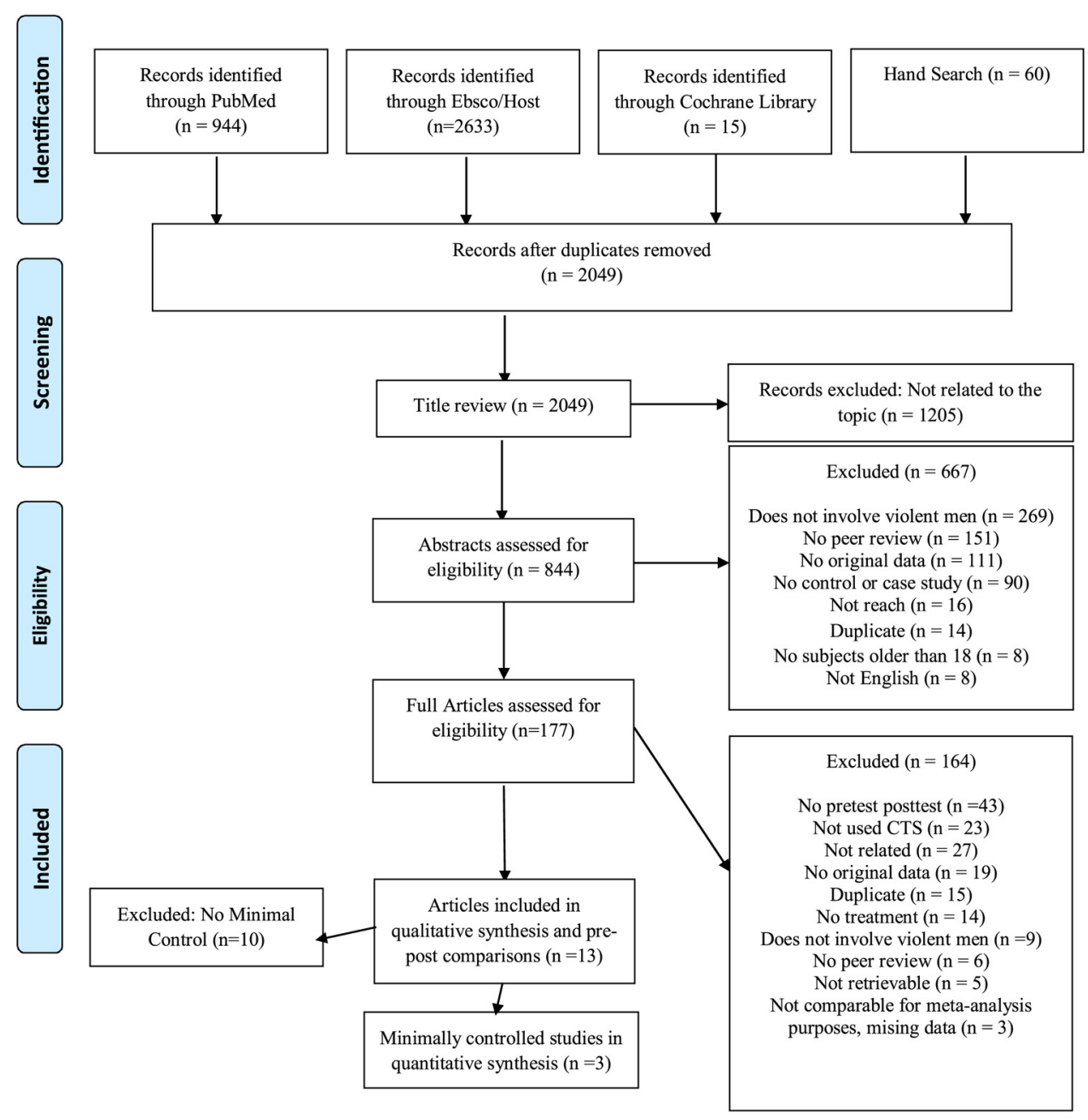

Fig. 1. PRISMA 2009 Flow Diagram for systematic review.

substance abuse component ( $\mathrm{md}=-2.45$, CI -4.12 to -0.77 ).

\subsection{Effects based on controlled studies: Minimal Control}

Of the 13 studies, only three were randomized intervention studies with minimal control groups. The forest plot for these studies is shown in Fig. 5 demonstrating mixed results. All three studies showed no significant differences between treatment and control although one study showed a suggestion of a difference which did not reach statistical significance. We provide a brief description of the 3 studies below. In 2011, Schumacher provided treatments based on Motivational Enhancement for physically abusive males who are participants of residential substance abuse treatment programs ( $\mathrm{md}=0.59$, CI -1.05 to 2.23). In the study by Taft et al (2015), they utilized a method called Strength at Home Men's Program (SAH-M) which is a cognitive behavioral group therapy that has a trauma focus (md $=-1.29$, CI -2.88 to 0.30 ). The program was designed for male veterans to decrease IPV in their relationship. Participants completed 12 weeks of group therapy. Compared to the enhanced treatment as usual (ETAU) condition, SAH$\mathrm{M}$ group reported significantly lower levels of physical and psychological violence. In the third study, Mbilinyi et al. (2011) tested the effectiveness of a Motivational Enhancement Therapy (MET) program for violent men with a history of substance abuse ( $\mathrm{md}=0.16$, CI -1.13 to 1.45). This study focused on investigating the group differences between males who participated in a personalized telephone-delivered MET versus receiving educational materials via mail but did not receive any personalized care.

\subsection{Effects based on head to head studies: Augmented Controls}

Two studies, Easton et al. (2007) and Stuart et al. (2013), compared the effectiveness of augmented substance abuse treatments to another active intervention to provide further information about the role of substance abuse treatments. One study supported the conclusion that augmented substance use components can have greater effects as found in the subgroup analyses of the pre-post studies while the other study did not. In particular, Easton 2007 investigated the effectiveness of a twelve-step substance (TSF) abuse program as compared to a cognitive behavioral Substance Abuse Domestic Violence (SADV) group in reducing violence and substance abuse over time. Seventy-eight men who were reported to police for domestic violence in the prior year were recruited for the study. At the end of the treatment, men in the SADV (based on CBT) group reported lower levels of substance abuse and violence as compared to the TSF group ( $\mathrm{md}=-6.02$, CI -6.99 to -5.05 ). Stuart et al. (2013), on the other hand, compared the effectiveness of Standard Batterer Intervention to Standard Batterer Program and Brief Alcohol Intervention for hazardously drinking men. The results were not as strong for this study (md $=1.6, \mathrm{CI}-1.91$ to 5.11 ) Table 2 .

\section{Discussion}

The current study was a systematic review and meta-analysis to understand the effectiveness of different batterer intervention programs that are designed for male perpetrators. Past research was inconclusive on showing the unique benefits of these programs. Many effect sizes of 


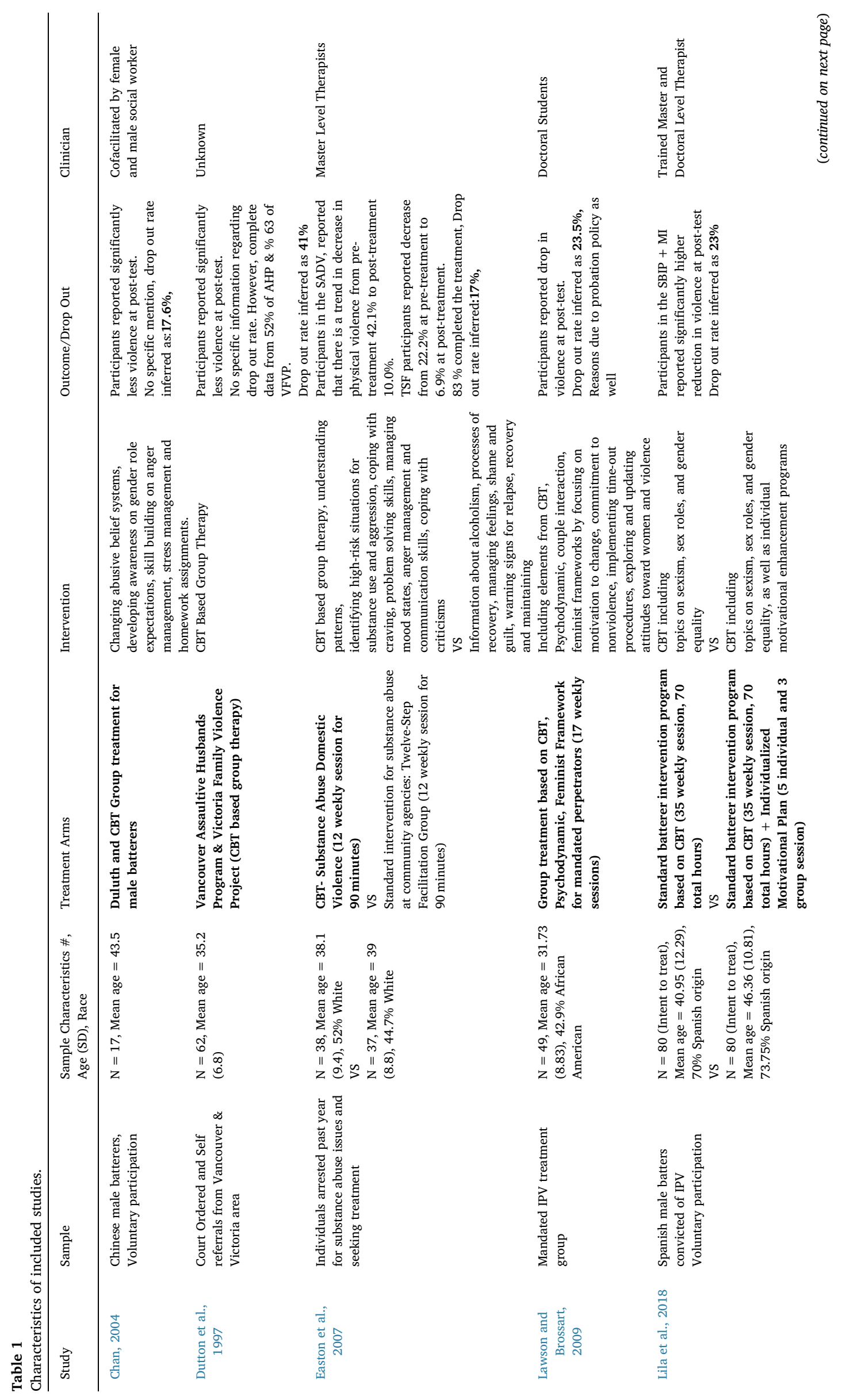




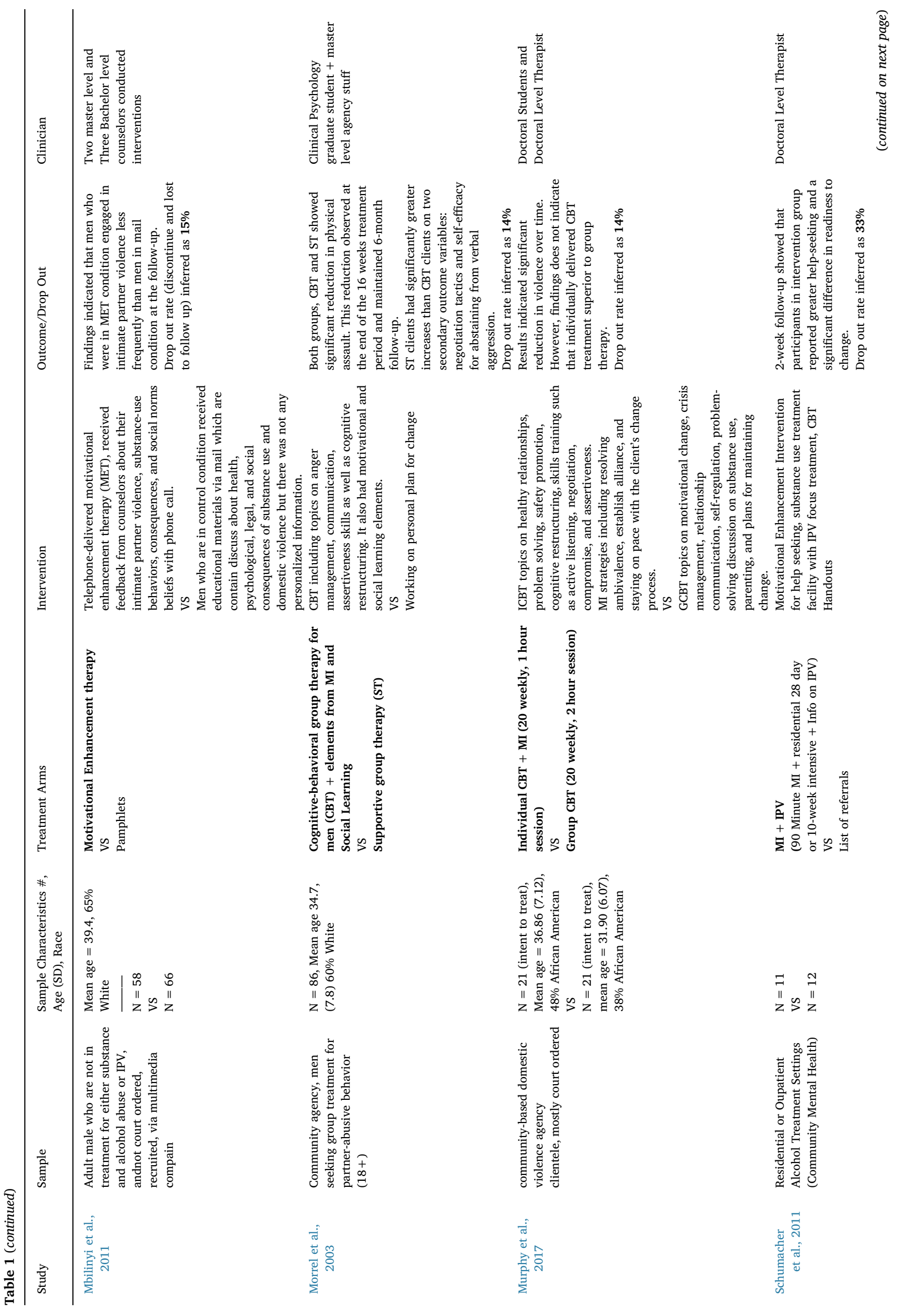




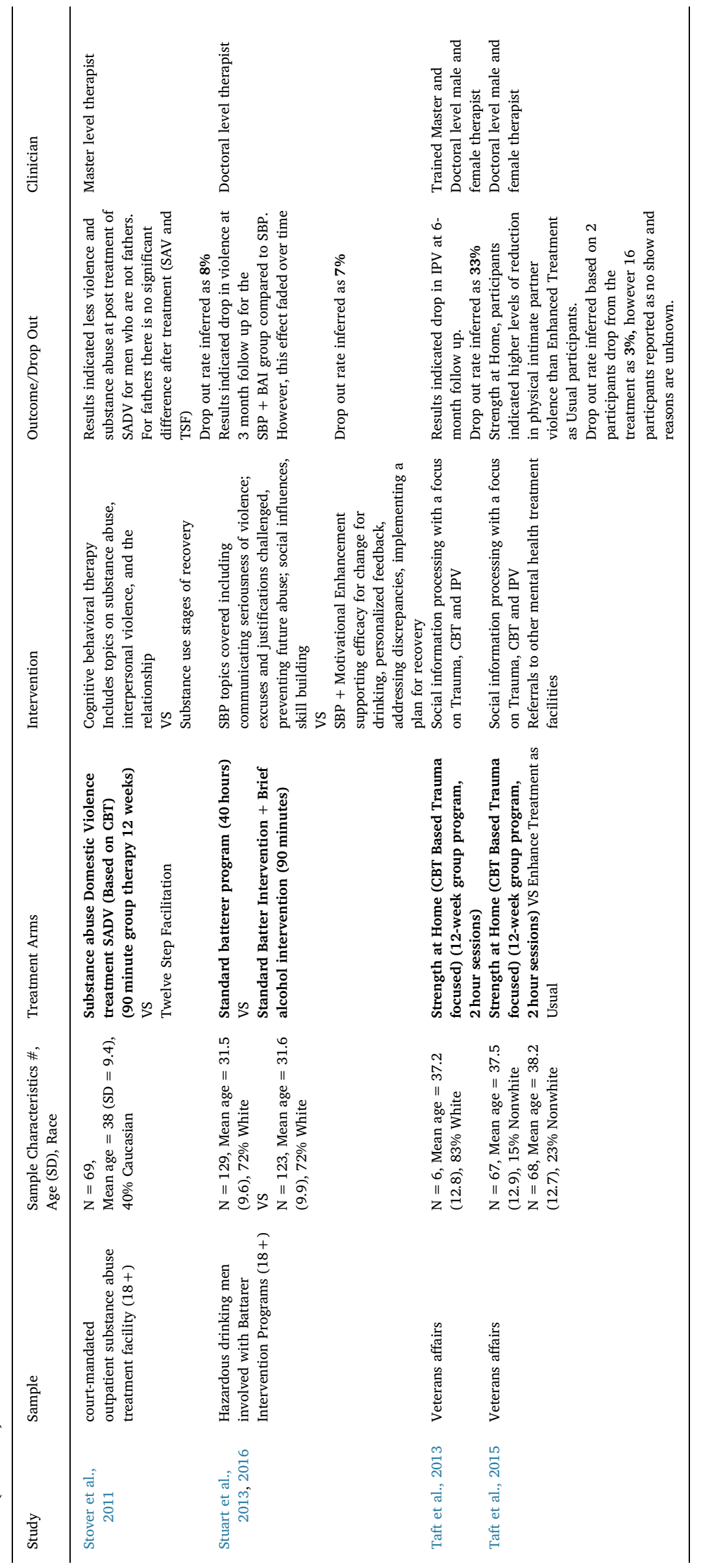



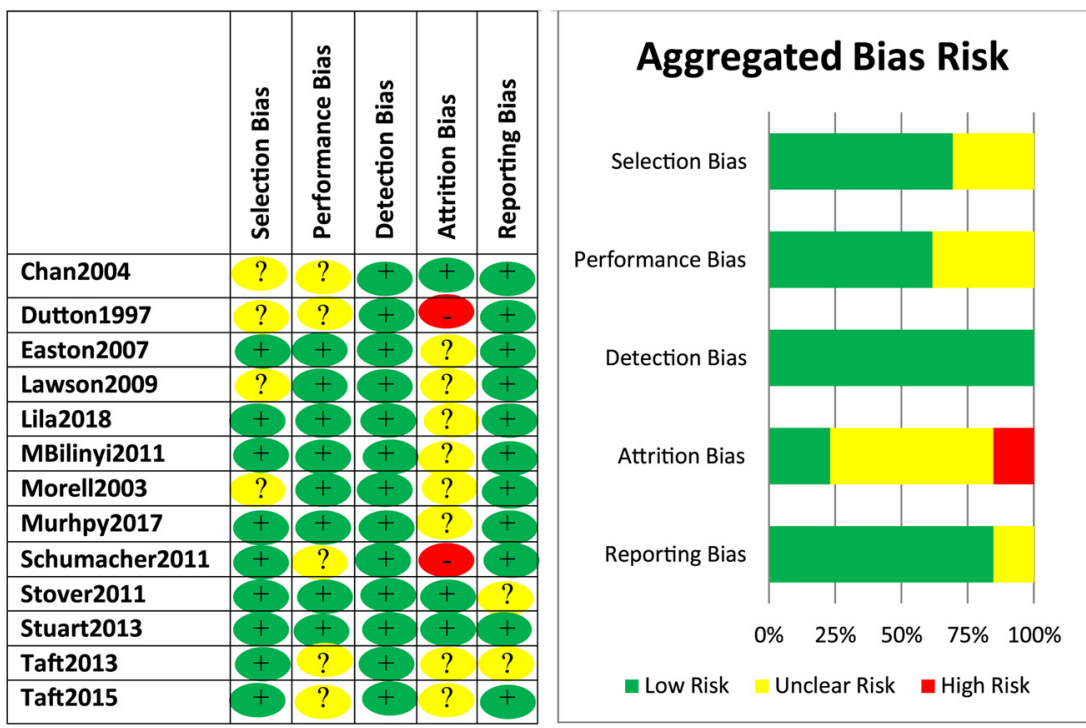

Fig. 2. Risk of Bias for Included Studies.

the included studies were small. Our examination of pooled pre and post data from 17 studies (including sub-studies) with 1492 participants (estimate $=-.85, \mathrm{SE}=.08, \mathrm{CI}-1.02$ to $-.69, \mathrm{z}=-10.28, \mathrm{p}<.001$ ) indicated that intervention programs that are designed for male perpetrators are an effective way to reduce violence at post-test among study samples. When conducting exploratory subgroup analyses, we observed that treatment approaches incorporating substance abuse and trauma yielded better results. Subgroup analysis also revealed that treatment models with sex roles components (Duluth models) yielded mixed results.

In comparison to the pre-post studies, the interventions in the three controlled studies with 223 total participants had no significant effects as compared to a minimal control group although one study reached borderline significance. These three studies were highly heterogeneous including a sample from residential treatment, veterans affair, and online treatment respectively. Lastly, the two head to head studies comparing substance abuse treatment and standard batterer intervention versus domestic violence treatment augmented with substance abuse treatment found that augmented treatments yielded mixed effects. Therefore, these head to head results do not support or refute the pre-post subgroup analyses showing potential benefits of substance abuse or trauma augmented interventions over other standard batterer interventions. Additional head to head studies will need to be conducted using these augmented approaches to determine their potential added benefit.

Standardized treatment approaches based on Duluth like frameworks that have curriculums including power and control dynamics, sex-role stereotyping, and gender-based values demonstrated mixed findings raising controversy about their effectiveness. Miller et al., 2013, in a recent meta-analysis, showed that participating in Duluth like models have no effect on violence reduction. Similarly, in this study, our results also show mixed results in the reduction of violence at post-test as compared to other treatments. This might be due to the directness of the intervention provided. While treatments based on CBT and MI, directly concentrating on diminishing violence by focusing on triggers and behavioral management strategies, treatment models based on Duluth and sex roles frameworks postulated that if perpetrators have a more egalitarian attitude about gender roles, they will behave less aggressively in the relationship. This indirect route may need a longer treatment time. It is also possible that the effect of sex role treatments might be more observable in treatment outcomes such as increased relationship satisfaction or increased egalitarianism in relationships.
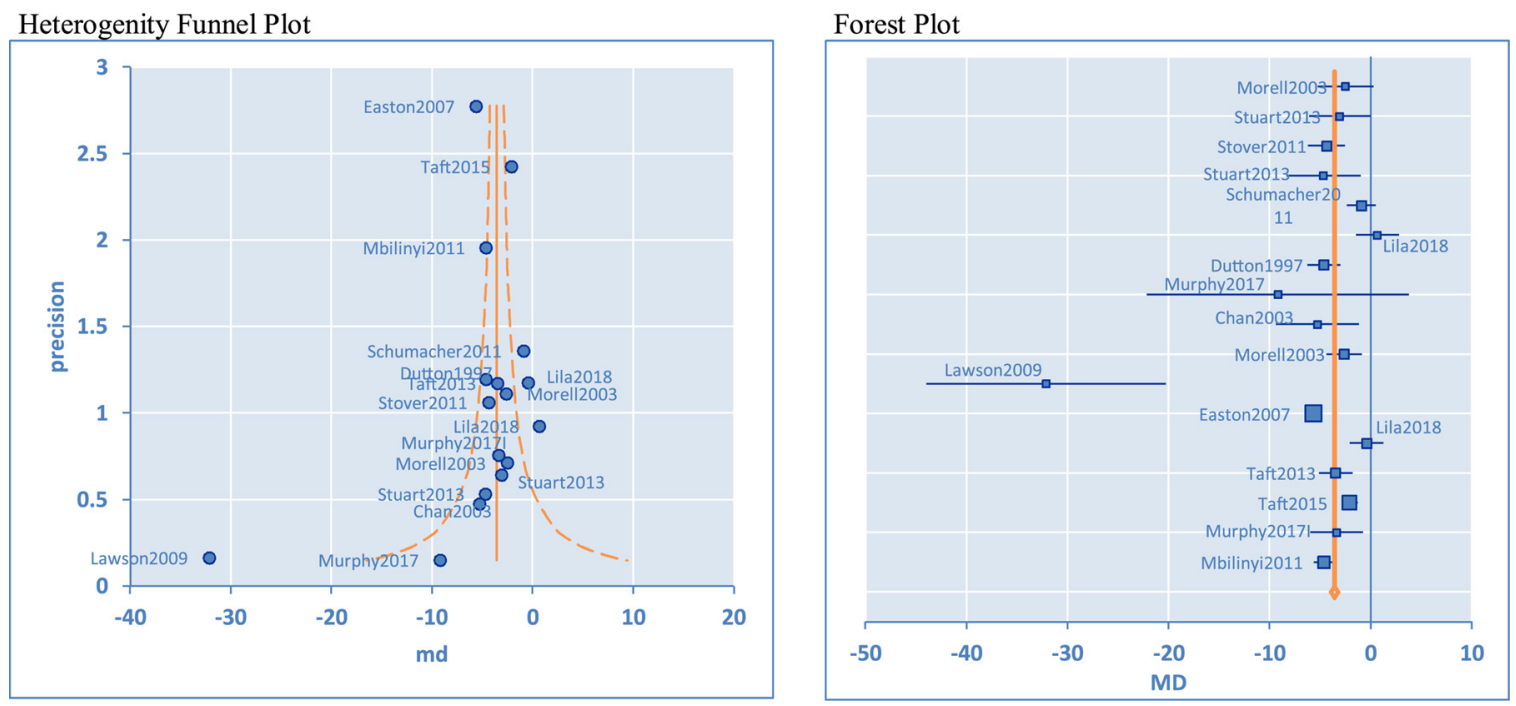

Fig. 3. Synthesis Results for Pre-Post Substudies. 


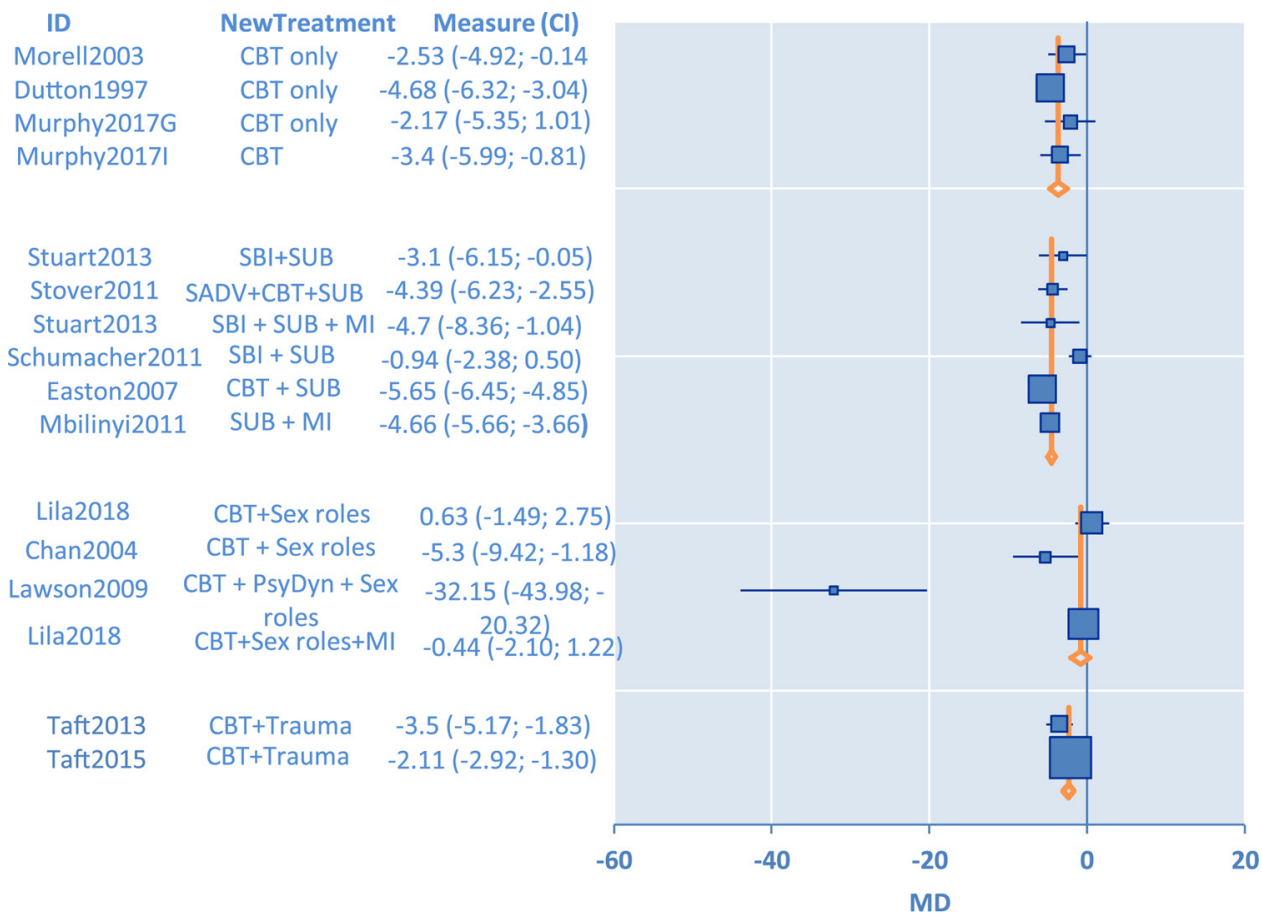

Fig. 4. Subgroup Forest Plot.

Lastly, studies including sex role components are mainly international studies (Hong Kong and Spain), and may be highly heterogeneous.

CBT for a partner abusive man usually includes interventions focusing on cognitive biases and psychoeducation on skill building such as assertiveness, emotional dysregulation, communication and problem solving (Wexler, 2013). Prior controlled studies on CBT revealed mixed results on the unique benefits of these interventions on reducing violence for male perpetrators. Studies compared the effectiveness of CBT to supportive group therapy (Morrel et al., 2003) and rigorous monitoring by case managers (Dunford, 2000a, 2000b) and process-psychodynamic treatment of painful childhood narratives (Saunders, 1996) were unable to show a significant benefit of $\mathrm{CBT}$ on reducing violence. Researchers concerned that inconsistent findings on CBT may be due to a lack of engagement in active intervention and inadequate collaboration (Alexander \& Morris, 2008; Murphy et al., 2017). In our study, we also observed that data pooled together from CBT only interventions were not significantly better in reducing violence as compared to other interventions based on motivational enhancement or standard treatments at post-test. This effect was similar for motivational techniques and the standard treatments. Many of the intervention methods have similar results. However, pre-post studies which used augmented substance abuse and trauma treatment yielded better results in reducing
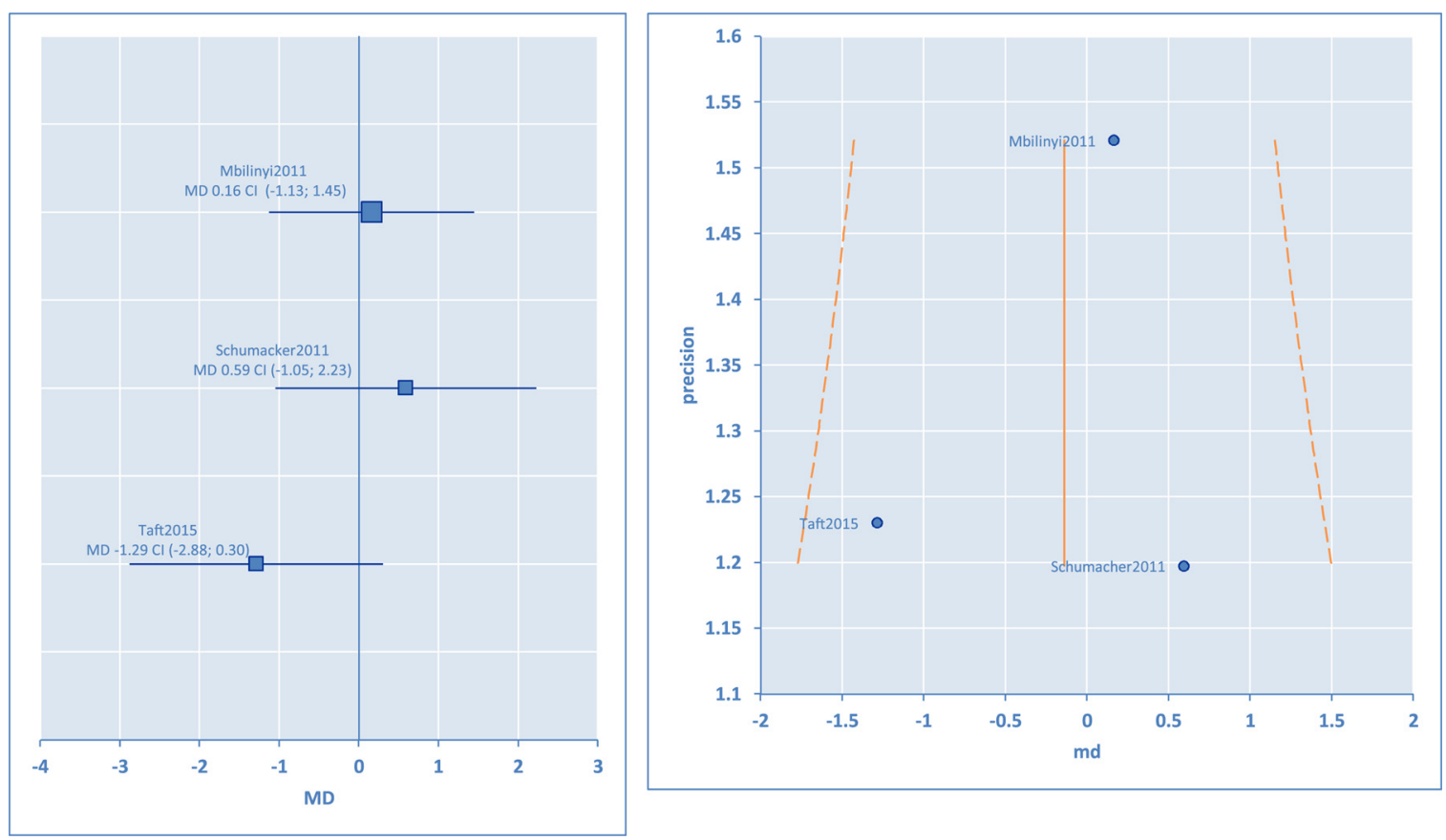

Fig. 5. Heterogeneity and Forest Plots for Minimally Controlled Studies. 
Table 2

Study subgroup comparisons

\begin{tabular}{|c|c|c|c|c|c|c|c|}
\hline \multirow{3}{*}{ Treatment } & \multirow{2}{*}{\multicolumn{2}{|c|}{ Studies Compared }} & \multicolumn{5}{|c|}{ Comparison } \\
\hline & & & \multirow[t]{2}{*}{ md } & \multirow[t]{2}{*}{ CI - } & \multirow[t]{2}{*}{$\mathrm{CI}+$} & \multirow[t]{2}{*}{$\mathrm{Z}$} & \multirow[t]{2}{*}{$\mathrm{P}$} \\
\hline & 1 & 2 & & & & & \\
\hline \multirow{9}{*}{$\begin{array}{c}\text { Substance Abuse } \\
\text { Augmented }\end{array}$} & $\mathrm{CBT}+\mathrm{SUB}$ & Chan2004; Dutton et al., 1997; Lawson and Brossart, 2009; Lila et al., 2018; Morrel & -2.14 & -3.20 & -1.08 & -3.96 & $<.001$ \\
\hline & Easton et al., 2007 & et al., 2003; Murphy et al., 2017; Taft et al., 2013, 2015 & & & & & \\
\hline & $\begin{array}{l}\text { Stover et al., } 2011 \\
\text { MI + SUB }\end{array}$ & & & & & & \\
\hline & Mbilinyi et al., 2011 & & & & & & \\
\hline & Stuart et al., 2013 & & & & & & \\
\hline & $\begin{array}{l}\text { Schumacher et al., } \\
2011\end{array}$ & & & & & & \\
\hline & $\mathrm{MI}+\mathrm{SUB}$ & CBT + SUB & 1.94 & 0.41 & 3.48 & 2.48 & $<.05$ \\
\hline & $\mathrm{CBT}+\mathrm{MI}$ & CBT + SUB & 3.83 & 1.89 & 5.77 & 3.86 & $<.01$ \\
\hline & CBT + SUB & CBT & -2.45 & -4.12 & -0.77 & -2.87 & $<.01$ \\
\hline Trauma Augmented & $\begin{array}{l}\text { Taft et al., } 2013 \\
\text { Taft et al., } 2015\end{array}$ & $\begin{array}{l}\text { Chan2004; Dutton et al., 1997, Easton et al., 2007; Murphy et al., 2017; Morrel } \\
\text { et al., 2003; Lawson and Brossart, 2009; Lila et al., 2018; Mbilinyi et al., 2011; } \\
\text { Stuart et al., 2013; Stover et al., 2011; Schumacher et al., } 2011\end{array}$ & -1.47 & -2.63 & -.30 & -2.47 & $<.001$ \\
\hline
\end{tabular}

the violence of perpetrators. Substance abuse and IPV are highly comorbid problems (Cunradi et al., 2014); therefore, addressing the underlying and urgent substance abuse problem at the outset seems to be yielding better results at reducing violence at post-tests. Similarly, recent research also indicates that IPV and trauma are highly comorbid (Rhodes et al., 2009). Treatment approaches augmented with a trauma component yielded improved results. It is possible that trauma taints how the participants' process information. Including trauma-based treatment components into the treatment might improve the processing of emotional, behavioral and cognitive pathways which may lead to violence. However, more research is needed to understand the mechanism for change, and whether this treatment works for diverse patients' groups since two studies involved veteran populations.

\subsection{Limitations}

Several limitations deserve mention and mainly focus on the limitations of the underlying literature being summarized. Many of the studies in the literature suffered from poor methodological rigor. One of the main limitations of this study was the lack of published studies with a control group for male perpetrators evaluating violence reduction. While pre-post studies showed a benefit of treatment, a control group with no intervention or an active comparator would help us determine the true benefit of treatment versus no treatment and the benefit of one treatment over another treatment. Therefore, the potential benefits found in the subgroup analyses of pre-post studies need further controlled studies to make clear delineations on which treatment would most benefit an individual. High attrition was also a limitation of the included studies. Attrition occurs frequently in domestic violence treatment populations and may lead to an overestimation of the benefit of the interventions if perpetrators who drop out are less likely to have decreased violence. However, the studies with lower attrition rates did have similar findings to the studies with higher attrition, making us more confident in the findings overall. Also, studies included in this meta-analysis are highly heterogeneous. To address this, we did use a random effects model to combine studies when appropriate and used subgroup analyses to further investigate areas of heterogeneity by intervention type. Once more studies are available to combine in metaanalyses, additional techniques can be used to identify potential sources of heterogeneity.

\subsection{Future Directions}

More experimental studies with comparison groups are needed to confirm and understand the role of treatment delivery in batterer intervention studies. Further research is also needed to explore the individual dynamics of these effective intervention studies since the heterogeneity of the included studies is relatively high. Our results indicate that treating underlying problems such as trauma and substance abuse as well as engaging participants in treatment over time is vital for success. These findings highlight the need for treatments that are augmented based on the needs of the participants

\subsection{Conclusion}

This study combines a systematic review with a meta-analysis and includes more updated research in terms of methodology and intervention strategies. Our exploratory results from pre-post data analyses suggest that targeted augmented approaches such as adding substance abuse or trauma components rather than generic approaches may work better for diminishing violence. Given the exploratory nature of these analyses and the lack of sufficient studies to fully explore other sources of heterogeneity and confounders, additional head to head studies of these and other novel approaches are critical if we want to decrease IPV within our communities.

\section{Author Note}

This publication was made possible by R01-LM012518 from the National Library of Medicine. Its contents are solely the responsibility of the authors and do not necessarily represent the official views of the NIH. There are no conflict of interests with study participants. This study involves already published research with aggregated data, it is not considered as human subjects research. Correspondence concerning this article should be addressed to Günnur Karakurt, Department of Psychiatry, Case Western Reserve University. Cleveland, OH 44106.

\section{References}

Adams, D., Cayouette, S., 2002. Emerge: A group education model for abusers. In: Adarondo, E., Mederos, F. (Eds.), Programs for men who batter: Intervention and prevention strategies in a diverse society. Civic Research Institute, Kingston 4-1, 4-32.

Alexander, P.C., Morris, E., 2008. Stages of change in batterers and their response to treatment. Violence and Victims 23, 476-492.

Armijo-Olivo, S., Stiles, C.R., Hagen, N.A., Biondo, P.D., Cummings, G.G., 2012 Assessment of study quality for systematic reviews: A comparison of the Cochrane Collaboration Risk of Bias Tool and the Effective Public Health Practice Project Quality Assessment Tool: Methodological research. Journal of evaluation in clinical practice 18 (1), 12-18.

Babcock, J.C., Canady, B.E., Graham, K., Schart, L., 2007. ). The evolution of battering interventions: From the dark ages into the scientific age. In: Hamel, J., Nicholls, T.L. (Eds.), Family interventions in domestic violence. Springer Publishing Company, New York, USA 215-244. 
Babcock, J.C., Green, C.E., Robie, C., 2004. Does batterers' treatment work? A metaanalytic review of domestic violence treatment. Clinical Psychology Review 23 (8), 1023-1053.

Babcock, J.C., La Taillade, J.L., 2000. Evaluating interventions for men who batter. Domestic violence: Guidelines for research-informed practice 33-77.

Bax, L., Yu, L.M., Ikeda, N., Tsuruta, H., Moons, K.G.M., 2006. Development and validation of MIX: comprehensive free software for meta-analysis of causal research data. BMC Medical Research Methodology 6, 50.

Black, M.C., Basile, K.C., Breiding, M.J., Smith, S.G., Walters, M.L., Merrick, M.T., Chen, J., Stevens, M.R., 2011. The National Intimate Partner and Sexual Violence Survey: 2010 Summary Report. . The Centers for Disease Control and Prevention.

Carney, M.M., Buttell, F.P., Muldoon, J., 2006. Predictors of batterer intervention program attrition: Developing and implementing logistic regression models. Journal of Offender Rehabilitation 43 (2), 35-54.

Chan, K.L., 2004. Group therapy for male batterers: a Chinese experience. Social Work with Groups 26 (4), 79-90.

Cunradi, C.B., Mair, C., Todd, M., 2014. Alcohol outlet density, drinking contexts and intimate partner violence: a review of environmental risk factors. Journal of drug education 44 (1-2), 19-33. https://doi.org/10.1177/0047237915573527.

DerSimonian, R., Laird, N., 1986. Meta-analysis in clinical trials. Controlled Clinical Trials 7, 177-188.

Dobash, R., Cavanagh, K., Lewis, R., 1996. Reeducation programs for violent men an evaluation. Research Findings 46, 14.

Dunford, F.W., 2000a. Determining Program Success: The Importance of Employing Experimental Research Designs. Crime \& Delinquency 46 (3), 425-434. https://doi. org $/ 10.1177 / 0011128700046003009$.

Dunford, F.W., 2000b. The San Diego Navy experiment: an assessment of interventions for men who assault their wives. Journal of Consulting and Clinical Psychology 68, 468-476.

Dutton, D.G., Bodnarchuk, M., Kropp, R., Hart, S.D., Ogloff, J.P., 1997. Client personality disorders affecting wife assault post-treatment recidivism. Violence and Victims 12 (1), 37-50.

Easton, C.J., Mandel, D.L., Hunkele, K.A., Nich, C., Rounsaville, B.J., Carroll, K.M., 2007. ). A cognitive behavioral therapy for alcohol-dependent domestic violence offenders: An integrated substance abuse-domestic violence treatment approach (SADV). American Journal on Addictions 16 (1), 24-31.

Feder, L., Wilson, D.B., 2005. A meta-analytic review of court-mandated batterer intervention programs: Can courts affect abusers' behavior? Journal of experimental Criminology 1 (2), 239-262.

Higgins, J.P.T., Green, S. (Eds.), 2011. Cochrane handbook for systematic reviews of interventions(5.1.0. ed.). The Cochrane Collaboration Retrieved February 05, 2017 from. www.cochrane-handbook.org.

Higgins, J.P., Thompson, S.G., Deeks, J.J., Altman, D.G., 2003. Measuring inconsistency in meta-analyses. BMJ: British Medical Journal 327 (7414), 557.

Gondolf, E.W., 2001. Batterer intervention systems: Issues, outcomes, and recommendations. Sage Publications.

Gondolf, E., 2004. Evaluating batterer counseling programs: A difficult task showing some effects and implications. Aggression and Violent Behavior 9, 605-631. https:// doi.org/10.1016/j.avb.2003.06.0.

Karakurt, G., Patel, V., Whiting, K., Koyuturk, M., 2016. Mining Electronic Health Records Data: Domestic Violence and Adverse Health Effects. Journal of Family Violence 29, 693-702.

Kelly, R., El-Sheikh, M., 2013. Longitudinal relations between marital aggression and children's sleep: The role of emotional insecurity. Journal of Family Psychology 27, 282-292. https://doi.org/10.1037/a0031896.

Lawson, D.M., Brossart, D.F., 2009. Attachment, interpersonal problems, and treatment outcome in group therapy for intimate partner violence. Psychology of Men \& Masculinity 10 (4), 288-301.

Lila, M., Gracia, E., Catalá-Miñana, A., 2018. Individualized motivational plans in batterer intervention programs: A randomized clinical trial. Journal of consulting and clinical psychology 86 (4), 309-320.

Mbilinyi, L.F., Neighbors, C., Walker, D.D., Roffman, R.A., Zegree, J., Edleson, J., O'Rourke, A., 2011. A telephone intervention for substance-using adult male perpetrators of intimate partner violence. Research on social work practice 21 (1), 43-56.

Miller, M., Drake, E., Nafziger, M., 2013. What works to reduce recidivism by domestic violence offenders? (Document No. 13-01-1201). Washington State Institute for Public Policy, Olympia.

Morrel, T.M., Elliott, J.D., Murphy, C.M., Taft, C.T., 2003. Cognitive Behavioral and Supportive Group Treatments for Partner-Violent Men. Behavior Therapy 34, 77-95.

Murphy, C.M., Meis, L.A., 2008. Individual Treatment of Intimate Partner Violence Perpetrators. Violence and Victims 23 (2), 173-186.

Murphy, C.M., Eckhardt, C.I., Clifford, J.M., Lamotte, A.D., Meis, L.A., 2017. Individual versus group cognitive-behavioral therapy for partner-violent men: A preliminary randomized trial. Journal of interpersonal violence. https://doi.org/10.1177/ 0886260517705666.

Pence, E., Paymar, M., 1993. Education groups for men who batter: The Duluth model. Springer Publishing Co., New York, NY, US.

Rhodes, K.V., Houry, D., Cerulli, C., Straus, H., Kaslow, N.J., McNutt, L.A., 2009. Intimate partner violence and comorbid mental health conditions among urban male patients. Annals of family medicine 7 (1), 47-55. https://doi.org/10.1370/afm.936.

Saunders, D.G., 2008. Group interventions for men who batter: A summary of program descriptions and research. Violence and Victim 23, 156-172.

Saunders, D.G., 1996. Feminist-cognitive-behavioral and process-psychodynamic treatments for men who batter: Interaction of abuser traits and treatment models. Violence and victims 11 (4), 393-414.

Schumacher, J.A., Coffey, S.F., Stasiewicz, P.R., Murphy, C.M., Leonard, K.E., FalsStewart, W., 2011. Development of a brief motivational enhancement intervention for intimate partner violence in alcohol treatment settings. Journal of Aggression, Maltreatment \& Trauma. 20 (2), 103-127.

Smith, S.G., Folwer, K.A., Niolon, P.H., 2014. Intimate partner homicide and corollary victims in 16 states: National Violent Death Reporting System, 2003-2009. American Journal of Public Health 104, 461-466.

Stover, C., Meadows, A., Kaufman, J., 2009. Interventions for intimate partner violence: Review and implications for evidence-based practice. Professional Psychology: Research and Practice 40, 223-233.

Stover, S.C., McMahon, T.J., Easton, C., 2011. The impact of fatherhood on treatment response for men with co-occurring alcohol dependence and intimate partner violence. The American Journal of Drug and Alcohol Abuse 37 (1), 74-78.

Stuart, G.L., McGeary, J., Shorey, R.C., Knopik, V.S., 2016. Genetics moderate alcohol and intimate partner violence treatment outcomes in a randomized controlled trial of hazardous drinking men in batterer intervention programs: A preliminary investigation. Journal of consulting and clinical psychology 84 (7), 592.

Stuart, G.L., Shorey, R.C., Moore, T.M., Ramsey, S.E., Kahler, C.W., O'farrell, T.J., et al, 2013. Randomized clinical trial examining the incremental efficacy of a 90-minute motivational alcohol intervention as an adjunct to standard batterer intervention for men. Addiction 108 (8), 1376-1384.

Taft, A.J., Hegarty, K.L., 2010. Intimate partner violence against women: what outcomes are meaningful? Jama 304 (5), 577-579.

Taft, C.T., Macdonald, A., Creech, S., Monson, C.M., Murphy, C.M., 2015. A Randomized Controlled Clinical Trial of the Strength at Home Men's Program for Partner Violence in Military Veterans. Journal of Clinical Psychiatry 77 (9), 1168-1175.

Taft, C.T., Macdonald, A., Monson, C.M., Walling, S.M., Resick, P.A., Murphy, C.M., 2013. "Strength at Home" group intervention for military populations engaging in intimate partner violence: Pilot findings. Journal of Family Violence 28 (3), 225-231.

Wathen, C.N., Macmillan, H.L., 2013. Children's exposure to intimate partner violence: Impacts and interventions. Pediatrics \& Child Health 18 (8), 419-422.

Wexler, D.B., 2013. The STOP domestic violence program: Group leader's manual.

von Hippel, P.T., 2015. The heterogeneity statistic $\mathrm{I}^{2}$ can be biased in small metaanalyses. BMC Medical Research Methodology 15, 35. https://doi.org/10.1186/s12874-0150024-z.

Yalom, I.D., Leszcz, M., 2005. The theory and practice of group psychotherapy. Basic Books, New York.

Zarling, A., Lawrence, E., Marchman, J., 2015. A randomized controlled trial of acceptance and commitment therapy for aggressive behavior. Journal of consulting and clinical psychology 83 (1), 199-212. https://doi.org/10.1037/a0037946. 